\title{
The role of gut microbiome in modulating response to immune checkpoint inhibitor therapy in cancer
}

\author{
Abdul Rafeh Naqash ${ }^{1}$, Alba J. Kihn-Alarcón ${ }^{2}$, Chara Stavraka $^{3}$, Kathleen Kerrigan ${ }^{4}$, \\ Saman Maleki Vareki ${ }^{5,6,7}$, David James Pinato ${ }^{8 \#}$, Sonam Puri ${ }^{9 \#}$ \\ ${ }^{1}$ Department of Investigational Cancer Therapeutics, National Cancer Institute, Bethesda, MD, USA; ${ }^{2}$ Department of Research, Liga Nacional \\ Contra el Cáncer \& Instituto de Cancerología, Guatemala City, Guatemala; ${ }^{3}$ Department of Medical Oncology, Guy's and St Thomas' NHS \\ Foundation Trust, Great Maze Pond, London, UK; ${ }^{4}$ Division of Medical Oncology Huntsman Cancer Institute at the University of Utah, Salt Lake \\ City, UT, USA; ${ }^{5}$ Division of Experimental Oncology, Department of Oncology, University of Western Ontario, London, ON, Canada; ${ }^{6}$ Department \\ of Pathology and Laboratory Medicine, University of Western Ontario, London, ON, Canada; ${ }^{7}$ Cancer Research Laboratory Program, Lawson \\ Health Research Institute, London, ON, Canada; ${ }^{8}$ Department of Surgery and Cancer, Imperial College, London, UK; ${ }^{9}$ Division of Medical \\ Oncology Huntsman Cancer Institute at the University of Utah, Salt Lake City, UT, USA \\ Contributions: (I) Conception and design: S Puri, DJ Pinato, AR Naqash; (II) Administrative support: None; (III) Provision of study material or \\ patients: None; (IV) Collection and assembly of data: All authors; (V) Data analysis and interpretation: None; (VI) Manuscript writing: All authors; \\ (VII) Final approval of manuscript: All authors. \\ \#These authors contributed equally to this work as senior authors. \\ Correspondence to: Sonam Puri. Division of Medical Oncology Huntsman Cancer Institute at the University of Utah, Salt Lake City, UT, USA. \\ Email: sonam.puri@hci.utah.edu.
}

\begin{abstract}
Immunotherapy has led to a paradigm shift in the treatment of several cancers. There have been significant efforts to identify biomarkers that can predict response and toxicities related to immune checkpoint inhibitor (ICPI) therapy. Despite these advances, it has been challenging to tease out why a subset of patients benefit more than others or why certain patients experience immune-related adverse events (irAEs). Although the immune-modulating properties of the human gut bacterial ecosystem are yet to be fully elucidated, there has been growing interest in evaluating the role of the gut microbiome in shaping the therapeutic response to cancer immunotherapy. Considerable research efforts are currently directed to utilizing metagenomic and metabolic profiling of stool microbiota in patients on ICPI-based therapies. Dysbiosis or loss of microbial diversity has been associated with a poor treatment response to ICPIs and worse survival outcomes in cancer patients. Emerging data have shown that certain bacterial strains, such as Faecalibacterium that confer sensitivity to ICPI, also have a higher propensity to increase the risk of irAEs. Additionally, the microbiome can modulate the local immune response at the intestinal interface and influence the trafficking of bacterial peptide primed T-cells distally, influencing the toxicity patterns to ICPI. Antibiotic or diet induced alterations in composition of the microbiome can also indirectly alter the production of certain bacterial metabolites such as deoxycholate and short chain fatty acids that can influence the anti-tumor tolerogenesis. Gaining sufficient understanding of the exact mechanisms underpinning the interplay between ICPI induced anti-tumor immunity and the immune modulatory role gut microbiome can be vital in identifying potential avenues of improving outcomes to cancer immunotherapy. In the current review, we have summarized and highlighted the key emerging data supporting the role of gut microbiome in regulating response to ICPIs in cancer.
\end{abstract}

Keywords: Gut microbiome; response; toxicity; immunotherapy

Submitted Sep 15, 2020. Accepted for publication Jan 12, 2021.

doi: $10.21037 /$ atm-20-6427

View this article at: http://dx.doi.org/10.21037/atm-20-6427 


\section{Introduction}

Immune checkpoint inhibitors (ICPIs) targeting programmed cell death protein (PD-1)/programmed cell death ligand (PD-L1) and cytotoxic T lymphocyteassociated protein (CTLA-4) axis revolutionized the management of cancer care with regulatory approvals in a variety of hematologic malignancies and solid tumors. These agents are approved as monotherapy, or in combination, and have shown improvements in objective response rate (ORR), progression-free survival (PFS), and overall survival (OS) compared to previous standard therapies. Additionally, they have a more favorable side effect profile than standard chemotherapy making them attractive therapeutic options in the first or later line settings. Unfortunately, only a minority of patients with cancer obtain a durable response to ICPIs, and the oncology community currently lacks a predictive biomarker across all malignancies to explain this phenomenon. At present, two predictive biomarkers, PD-L1 immunohistochemistry (IHC) and tumor mutation burden $(\mathrm{TMB})$ are utilized in clinical practice, although TMB has demonstrated mixed results as an effective predictive biomarker $(1,2)$. Additionally, while PD-L1 IHC has demonstrative efficacy as a predictive biomarker particularly in non-small cell lung cancer (NSCLC) and other solid tumors, there are numerous testing platforms with companion diagnostic status, differing positive cutoffs among malignancies, and several interpretive scoring systems which can create confusion among clinicians.

More recently, there has been growing interest in the tumor microenvironment (TME) and in the gut microbiome as potential predictive biomarkers to ICPIs. The gut microbiome has been extensively studied and found to play a significant role in human health and in the pathogenesis of chronic diseases such as cancer, the metabolic syndrome, and diabetes mellitus $(3,4)$. Retrospective clinical data suggests that antibiotic use prior to ICPI can reduce the effectiveness of these agents, likely through altered composition of the gut microbiome (5). A succession of clinical studies has shown that responders (R) to ICPIs can be differentiated from non-responders (NR) based on the composition of their pretreatment gut microbiota $(6,7)$. Emerging data have shown that certain bacterial strains confer sensitivity to therapy and protection against toxicity while others increase the risk of immunerelated adverse events (irAEs) (8). Although early studies primarily used mice models, there is now mounting data from human cohorts, suggesting that the gut microbiota is a dominant force in mediating both response and toxicity to these therapeutic strategies (9).

In the current review, we will summarize the emerging data supporting the role of the gut microbiome as a predictive biomarker of response and toxicity to ICPI therapy.

\section{Gut microbiome and response to ICPIs}

The human intestine accommodates over a trillion of commensal microorganisms which exist in a symbiotic relationship with their host and constitute the human gut microbiota (10). A number of studies have demonstrated that compositional alterations in the gut microbiome can promote tumorigenesis as well as having a detrimental effect to ICPIs responses via immune modulatory properties (11-14). Loss of microbial diversity (also known as dysbiosis) has been associated with poor treatment responses to ICPIs and worse survival outcomes in cancer patients (11). On the contrary, enrichment of the gut in certain bacterial species such as Faecalibacterium, Bifidobacterium, Lactobacillus, Akkermansia muciniphila, and Ruminococcaceae spp. has been found to have a favorable impact on ICPI response $(8,15,16)$. Considerable research efforts are currently directed to the clinical utilization of metagenomic and/ or metabolic profiling of patients' stool microbiota as a potential biomarker of responsiveness to ICPI to be utilized in clinical trials and in routine practice.

\section{Putative mechanisms underlying the relationship between gut microbiome and response to ICPI}

The immune modulating properties of the gut bacterial ecosystem are yet to be fully elucidated. A number of mechanisms have been identified involving suppression of pro-inflammatory cytokines, reduction of T-regulatory cells (Tregs) density (8), stimulation of anti-tumor dendritic cell maturation and accumulation of antigenspecific T-cells in the TME (15) (Figure 1). Modulation of major histocompatibility complex class I/II genes, with increased T-cell recognition of cancer cells is amongst the key mechanisms postulated to underlie the positive relationship between a physiologic gut microbial state and response to immunotherapy (17). More recent evidence has emphasized the significance of imbalance in microbial metabolites as an additional and non-mutually exclusive mechanism governing the immune homeostasis of the gut 


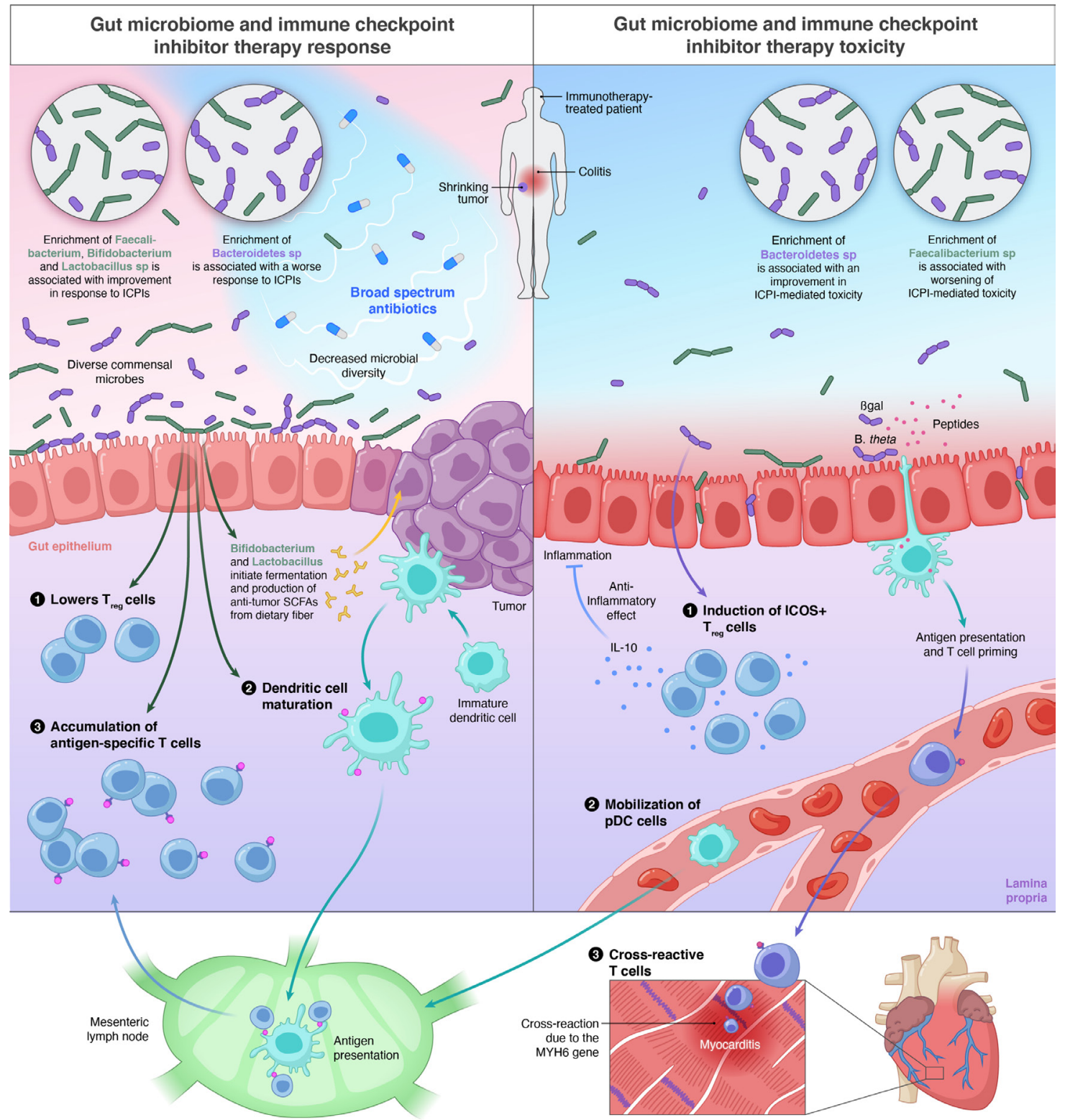

Figure 1 The role of gut microbiome in regulating response to ICPI. Right: Gut microbiome and ICPI response. Enrichment of certain gut bacteria are associated with improvement in response (Faecalibacterium, Bifidobacterium and Lactobacillus sp) or worse response (Bacteroides sp) to ICPIs. Possible mechanisms of ICPI response modulation include lowering of T regulatory cells (Treg), stimulation of tumor-specific dendritic cell maturation or accumulation of antigen-specific $T$ cells. The use of antibiotic therapy decreases the diversity of gut microbiome and is associated with adverse response rates and survival in patients on ICPIs. Certain bacterial species (Bifidobacterium and Lactobacillus) enhance fermentation of dietary fiber to form anti-tumor SCFAs. Left: Gut microbiome and ICPI toxicity. Enrichment of certain bacteria are associated with higher irAEs (Faecalibacterium sp) and lower irAEs (Bacteroides $s p$ ). Possible mechanisms of ICPI toxicity modulation include proliferation of ICOS + Tregs that secrete IL-10 (an anti-inflammatory cytokine) in the colonic lamina propria and mobilization of pDC. Peptides derived from certain bacteria that can also induce cross antigen T-cell reactivity leading to irAEs at distant sites. In this case B-gal peptides are taken up by the APC and lead to generation of heart-specific T-cells that cross react against MYH6 on the cardiac muscle leading to myocarditis. ICPI, immune checkpoint inhibitor; SFCA, short chain Fatty acids; Treg, T regulatory cell; irAEs, immune related adverse events; ICOS, inducible T cell co-stimulator; Pdc, plasmacytoid dendritic cell; APC, antigen presenting cells; IL-10, interleukin 10; B-gal, beta galectin; MYH6, Myosin Heavy Chain 6. 
mucosa. For instance, the relative under-representation of short chain fatty acids produced by bacterial species known to be positively associated with response to ICPIs such as Akkermansia spp. Enterococcus spp. and Faecalibacteria, and an enhanced conversion of primary to secondary bile acids such as deoxycholate by Clostridiales might explain the relative functional contribution of the diverse bacterial taxa in shaping anti-tumor tolerogenesis (18).

\section{Antibiotic mediated modulation of gut microbiome and response to ICPIs}

Iatrogenic factors are an important source of variation in the gut microbiome. Over the years, there has been an increased appreciation of how concomitant medications that are commonly prescribed in patients with cancer may affect response to immunotherapy (19). Broad-spectrum antibiotics (ATB) are widely used in cancer patients, a patient population at higher risk of bacterial infections due to treatment or disease-related immune suppression. Despite the significance of ATB in reducing infectionrelated mortality, their administration even in short courses causes detrimental and protracted changes in the microbiota disturbing the symbiotic equilibrium with the host (20). This class of drugs has therefore been brought to the forefront of research for their impact on the efficacy of ICPIs therapeutics.

There is a growing body of evidence associating the use of ATB with adverse response rates and survival outcomes in patients receiving ICPIs across a range of solid tumors (21-29). It is of note that a similar effect has not been observed for narrow spectrum antibiotics in the existing literature, though with less available data (23). This is likely attributed to the greater impact of broad-spectrum antibiotics in disrupting the taxonomic composition of the gut microbiota. Timing of antibiotic treatment was identified as an independent factor heavily impacting ICPI response. A study by Pinato et al. revealed that administration of ATB up to 30 days prior to commencing ICPIs in patients with a variety of solid tumors resulted in significantly shorter OS compared to concurrent administration (2 vs. 26 months respectively) (21). Apart from ATB treatment timing, duration also affects response to ICPIs. In their study, Tinsley et al. demonstrated that multiple ATB courses or a single prolonged course of more than 7 days, had the worst overall PFS (median PFS, 2.8 months; HR, 2.625; $\mathrm{P}=0.026$ ) and $\mathrm{OS}$ outcomes (median OS, 6.3 months; HR, 1.904; $\mathrm{P}=0.009$ ) (22).
The potential role of the human gut microbiome in modulating response to ICPI has generated considerable interest around the development of microbiome-based therapies. A number of early phase clinical trials are underway exploring the ability of microbial therapies to augment the efficacy of ICPIs in advanced solid malignancies. These novel therapies are proposed to introduce selected bacterial species known to positively influence anti-tumor immunity or restore gut microbiome diversity to a physiological state. A summary is provided in Table 1.

Gaining sufficient understanding of the exact mechanisms underpinning the interplay between immune cells and microorganisms which mediates the adverse relationship between ATB and ICPI response is crucial for the development of therapeutic interventions and warrants further research. Such interventions would aim at preventing the disturbance or restoring the microbiome homeostasis in the form of dietary interventions with pre- or pro-biotics, ingestion of certain bacterial species, selective antibiotic treatment or fecal microbial transplantation (FMT) (30). As ATB will remain the cornerstone of infection treatment in the cancer patients, combinational approaches involving rationalization of ATB use in patients receiving ICPIs along with interventions to maintain microbiome homeostasis will be crucial to not compromise treatment efficacy. Furthermore, some preclinical and clinical evidence have shown a carcinogenic effect for certain bacterial species in pancreatic cancer via immune suppression mechanisms. Antibiotic ablation of harmful bacteria re-established antitumor immunity and sensitized pancreatic tumors to ICPIs in the animal models (31). Furthermore, a short course of antibiotic treatment before receiving FMT could, in theory, enhance donor microbiome engraftment in the host; however, a preclinical study of transplanting stool from $\mathrm{BALB} / \mathrm{c}$ mice into C57BL/6 mice only showed a minor effect on engraftment (32). As we are learning more about the positive and negative immune modulating role of gut and tumor microbiome, antibiotics could be used to reduce harmful bacteria as we are moving more towards testing patient's microbiome before the start of treatment.

\section{Gut microbiome and toxicity to ICPIs}

ICPIs have had tremendous success in the treatment of various malignancies resulting in sustained responses. However, a proportion of patients continue to experience treatment-limiting toxicities that are termed as irAEs (7). 
Table 1 Trials evaluating microbiome modulation in patients with advanced malignancies

\begin{tabular}{|c|c|c|c|c|}
\hline Trial & Patient population & Intervention & Outcome & Status \\
\hline $\begin{array}{l}\text { (NCT03817125) } \\
\text { Phase I }\end{array}$ & Metastatic melanoma & $\begin{array}{l}\text { Donor-derived live bacteria } \\
\text { composition (SER-401) } \\
\text { dosed in combination with } \\
\text { nivolumab, after vancomycin } \\
\text { pretreatment }\end{array}$ & $\begin{array}{l}\text { Primary: frequency of AEs; secondary: } \\
\text { determination of SER- } 401 \text { bacteria } \\
\text { engraftment; ORR; DCR; PFS; OS; } \\
\text { duration of response; change in the } \\
\text { percentage of CD } 8 \text { cells in tumor tissue } \\
\text { from baseline at Cycle } 2\end{array}$ & $\begin{array}{l}\text { Currently } \\
\text { recruiting }\end{array}$ \\
\hline $\begin{array}{l}\text { (NCT04193904) } \\
\text { Phase I }\end{array}$ & $\begin{array}{l}\text { Resectable pancreatic } \\
\text { adenocarcinoma }\end{array}$ & $\begin{array}{l}\text { Oral live biotherapeutic } \\
\text { MRx0518 with } \\
\text { hypofractionated } \\
\text { preoperative radiation for } \\
\text { resectable pancreatic cancer }\end{array}$ & $\begin{array}{l}\text { Primary: safety of MRx0518 in } \\
\text { combination with hypofractionated } \\
\text { preoperative radiation; secondary: major } \\
\text { pathologic response; change in TILS; OS; } \\
\text { PFS; local control; distal control }\end{array}$ & Recruiting \\
\hline $\begin{array}{l}\text { (NCT03637803) } \\
\text { phase I/II }\end{array}$ & $\begin{array}{l}\text { Advanced and/or metastatic or } \\
\text { recurrent; non-small cell lung } \\
\text { cancer, renal cell carcinoma, } \\
\text { bladder cancer or melanoma }\end{array}$ & $\begin{array}{l}\text { MRx0518 In Combination } \\
\text { With pembrolizumab }\end{array}$ & $\begin{array}{l}\text { Primary: safety and tolerability } \\
\text { of MRx0518 in combination with } \\
\text { pembrolizumab; clinical benefit; } \\
\text { secondary: anti-tumor effect with imaging }\end{array}$ & Recruiting \\
\hline $\begin{array}{l}\text { (NCT02928523) } \\
\text { Phase II }\end{array}$ & $\begin{array}{l}\text { Acute myeloid leukemia \& } \\
\text { high-risk myelodysplastic } \\
\text { syndrome }\end{array}$ & $\begin{array}{l}\text { Autologous Fecal Microbiota } \\
\text { Transplantation }\end{array}$ & $\begin{array}{l}\text { Primary: evaluation of AFMT efficacy in } \\
\text { dysbiosis correction; evaluation of AFMT } \\
\text { efficacy in MDRB eradication; secondary: } \\
\text { definition of a dysbiosis biosignature }\end{array}$ & Completed \\
\hline $\begin{array}{l}\text { (NCT04167137) } \\
\text { Phase I }\end{array}$ & $\begin{array}{l}\text { Stage III or IV solid tumor or } \\
\text { lymphoma (inoperable) }\end{array}$ & $\begin{array}{l}\text { Synthetic biotics (SYN1B891) } \\
\text { dosed in combination with } \\
\text { atezolizumab }\end{array}$ & $\begin{array}{l}\text { Primary: incidence of DLTs; secondary: } \\
\text { nature, incidence, and severity of all AEs } \\
\text { and SAEs; ORR }\end{array}$ & Recruiting \\
\hline
\end{tabular}

AEs, adverse event; SAE, serious adverse event; PFS, progression free survival; ORR, objective response rate; DCR, disease control rate; PFS, progression free survival; OS, overall survival; TILS, tumor infiltrating lymphocytes; DLT, dose-limiting toxicity; AFMT, autologous fecal microbiota transplantation; MDRB, multidrug resistant bacteria.

The heterogeneity of patient responses, as is commonly witnessed with ICPIs, extends to toxicity as well. The variable irAEs associated with ICPI therapy commonly include those of dermatological, gastrointestinal, pulmonary, hepatic and endocrine origin, as well as less frequent immune-toxicities such as ocular, type 1 diabetes mellitus, cardiac, neurological, and hematological origin. These irAEs have the potential to complicate the management of patients with cancer, often leading to discontinuation of treatment, which may ultimately influence outcomes (33). There is mounting preclinical and clinical data highlighting the potential influence of gut microbiome on irAEs. Additionally, the role of specific microbiota signatures and their interaction with other factors that produce dysbiosis (i.e., antibiotic use, aging, and obesity) is an area of active investigation (34-36).

\section{Potential mechanisms between irAEs onset and microbiome characterization}

ICPIs act by removing the inhibitory checkpoints on immune cells to activate effector T-cells to target tumors. However, ICPI-induced T-cell cross-reactivity between normal and tumor tissue tends to cause irAEs (37). The most common sites of adverse effects include the gut and skin, which are characterized by rapid cellular turnover and have a close association with bacteria, suggesting a role of the microbiota in toxicity. Although evidence indicates a strong association between microbiota and the immune system, the exact mechanism mediating this toxicity is unclear. It is also believed that the identification of microbial signatures could serve as biomarkers, and thus may help in the development of microbial-based therapeutics and 
strategies to identify patients at risk of inflammatory complications caused by cancer immunotherapy (38).

To date, most of the data relating to gut microbiome and irAEs has been in the context of immune-mediated colitis and anti-CTLA-4 treatment. In preclinical murine models, Vétizou et al. provided the first evidence of a potential gut microbiome dependent mechanism underlying ICPI-associated toxicity. In this study the authors found that anti-CTLA-4 mAb induced a "subclinical colitis" that is dependent on the gut microbiota, which was more prominent in mice kept in specific-pathogen-free (SPF) conditions than in germ-free (GF) animals (39). They observed that cell death and the proliferation of intraepithelial cells (IECs) were increased in the ileum and colon of the SPF mice after the first dose of anti-CTLA-4 $\mathrm{mAb}$, an effect that was also observed after adding toll-like receptor (TLR) agonists (microbial ligands in this assay). Furthermore, they demonstrated the potential role of intraepithelial lymphocytes (IELs) in ICPI-induced colitis by administrating antibodies against TLRs which abolished the apoptosis and proliferation of IECs (39). The authors demonstrated an improvement in the histopathological findings of colitis in anti-CTLA-4-treated mice with oral gavage of B. fragilis and Burkholderia cepacia (39), and concluded that the protective effect of $B$. fragilis on immunemediated colitis could be related to its ability to promote the proliferation of ICOS+ Tregs that secrete IL-10 (an anti-inflammatory cytokine) in the lamina propria. Another possible mechanism is believed to be through gut microbiota induced mobilization of plasmacytoid dendritic cells (DCs) that have been observed to accumulate and mature in mesenteric lymph nodes after B. fragilis monocolonization of GF mice treated with antiCTLA-4 mAb $(39,40)$. In addition, the anti-inflammatory effect of gut microbiota produced by the stimulation of Treg cell differentiation has also been proposed in a prospective study by Dubin et al. in patients with ICPImediated colitis where the authors found that bacteria from the Bacteroidetes phylum family (Bacteroidaceae, Rikenellaceae, and Barnesiellaceae), that form a major phyla of human gut microbes were enriched in colitis-resistant patients (41).

In contrast to the protective effect of the Bacteroidetes phylum family, a study by Chaput et al. concluded that the baseline enrichment of Faecalibacterium and other Firmicutes bacteria was not only associated with an increased likelihood of ipilimumab-induced colitis but also associated with an improvement in survival on CTLA-4 blockade. Their data elucidated a higher representation of Bacteroidetes in patients with poor anti-tumor responses and decreased incidence of colitis, which align with findings from Vetizou $e t a l$. and Dubin et al. $(8,39,41)$. Additionally, to further elucidate the balance between ICPI response and toxicity the authors also performed flow cytometry on whole blood at baseline and correlated immune populations with microbiota composition. Their findings indicated that development of immune-mediated colitis was associated with at least a two-fold reduction in the Firmicutes phylum family of bacteria (Ruminococcus, Lachnospiracea incertae sedis, Blautia, Clostridium, Eubacterium, unclassified Lachnospiraceae, and Pseudoflavonifractor) that were dominant members of the microbiota at baseline levels. Interestingly, patients that had evidence of immune-mediated colitis also showed a decreased baseline levels of blood pro-inflammatory cytokines IL-6, IL-8, and sCD-25 compared to those who did not develop colitis indicating the potential interaction of systemic inflammatory proteins and the gut microbiome composition. Furthermore, they observed an association between presence of Faecalibacterium and low baseline percentage of circulating $\alpha 4^{+} \beta 7^{+}$T-cells and $\mathrm{CD} 4^{+}$Tregs raising the possibility of this being a factor influencing better responses to ICPIs and higher incidence of colitis (8). There is also evidence to suggest a potential role of pretreatment microbiome and response to ICPIs. In a study, Simpson et al. analyzed 38 stage III melanoma patients' pretreatment fecal microbiomes and found that low microbial diversity and a reduction in the abundance of butyrate-producing Ruminococcaceae and methanogenicarchaea were associated with a lack of response to ICPIs and the development of severe irAEs. They also found that differences in peripheral immune cells were associated with changes in microbial diversity (42). The contradictory observations between Simpson et al. study and Chaput et al. study on the incidence of ir-AEs and the association with response to ICPIs, hints at a different role that bacterial diversity and enrichment of certain strains of bacteria can play in conferring sensitivity to treatment and potentially inducing ir-AEs. Given the variations that exist in gut microbiome in patients from different geographic areas, more studies are required to confirm these observations and help us better understand the independent role of gut microbiome diversity and certain families of bacteria in treatment response and toxicity in patients that receive ICPIs.

Interestingly, the microbiome mediation of irAEs is not only limited to colitis. A recent study by Gil-Cruz 
et al. demonstrated the influence of microbiota as an important internal environmental influence driving lethal myocarditis. They used a modified transgenic murine model expressing a myosin heavy chain 6 (MYH6)- specific T-cell receptor on more than $95 \%$ of their CD4+ T-cells (TCRM) to evaluate how heart-specific T-cells cross-react with microbial components resulting in myocarditis. They showed that CD4+ TCRM cells that had infiltrated the heart expressed significantly higher gut-homing receptors under SPF conditions compared with GF conditions, indicating a transfer of TCRM T-cells that initially proliferated in the lamina propria of the colonic tissue to the mediastinal lymph node and finally to the cardiac tissue. More interestingly, they demonstrated peptides derived from Bacteroides thetaiotaomicron (B. theta) and Bacteroides faecis $\beta$-galactosidase ( $\beta$ gal) being similar to MYH6 thereby highlighting the potential pathological role of crossreactive T-cells that proliferate in the gut lamina propria and eventually lead to myocarditis. In their study, transgene negative and TCRM mice possessed disparate microbiomes and its modification by antibiotic treatment prevented lethal cardiomyopathy and reduced cardiac inflammation. These findings provide insight on the mechanism on how gut microbiome could potentially increase the likelihood of cardiotoxicity in patients undergoing ICPIs (43).

Lastly, there is also data to suggest a potential role of dysbiosis produced by other factors affects response and toxicity to ICPIs. A recent study by Mohiuddin et al. demonstrated that antibiotic-induced dysbiosis increases the likelihood of grade 4 immune-mediated colitis in melanoma patients treated with ICPIs needing treatment with steroids and is related to worse OS among these patients (44).

\section{Manipulation of the microbiome to reduce irAEs}

As previously mentioned, Dubin et al. have demonstrated a correlation between over-representation of the Bacteroidetes phylum and resistance to immune-mediated colitis in humans (41). In line with these findings, a cocktail of Bacteroidales and Burkbolderiales was seen to ameliorate CTLA-4-blockade-induced subclinical colitis and colon inflammatory scores in antibiotic-treated mice. Additionally, this cocktail was also observed to influence the anti-tumor efficacy of CTLA-4 blockade via T helper type $1\left(\mathrm{~T}_{\mathrm{h}} 1\right)$ immune response and maturation of intra tumoral DCs (39). Another study by Wang et al. [2017] also using a murine model demonstrated the use of microbiota manipulation by administrating four Bifidobacterium species using oral gavage that ameliorated ICPI-associated colitis in the mice (45).

Wang et al. [2018] reported the first series of cases of ICPI-associated refractory colitis successfully treated with FMT, providing preliminary evidence to support modulation of the gut microbiome to treat ICPI-related colitis (46). In addition to an endoscopic resolution of colitis, the authors showed a durable change in the gut microbiota of the recipient resembling that of the donor, and an alteration in the colonic inflammatory infiltrate to a more anti-inflammatory phenotype following FMT. Additional studies are critical to assess the utility of this approach as well as to provide further mechanistic insights (12) $(9,47,48)$. Key clinical trials that are currently underway and evaluating this approach are listed in Table 2.

Recently it has been identified that the loss of control over self- and cross-reactive T-cells during ICPI therapy may be a reason for potentially lethal cardiac inflammation in patients who share particular HLADQA1*/B1* alleles. This was shown to be linked to bacterial peptides derived from B.Theta, B.faeces that demonstrated the ability to activate heart-specific T-cells cross-reacting against MYH6. Thus, targeting the microbiome of genetically predisposed myocarditis patients or susceptible patients undergoing ICPI treatment through antibiotics to reduce bacteriainduced immunoreactivity may alleviate disease severity and may, therefore, help prevent the potentially lethal sequelae of inflammatory cardiomyopathy (43). This might be even true for other irAEs, where perhaps a similar role of bacteria-induced immunoreactivity needs to be ascertained, with the ultimate aim of modifying these host-specific factors to achieve improved patient outcomes.

Efforts to characterize gut microbiota that contribute to toxicity to immune checkpoint blockade are underway (Table 1), the trial [NCT04107168] will be evaluating microbiome immunotherapy toxicity and response in 9 cohorts of patients with melanoma, renal cell carcinoma and NSCLC receiving different ICPIs therapies. Other studies will be evaluating the composition of the fecal microbiome and its correlation with irAEs in patients with melanoma and lung cancer [NCT03643289, NCT03688347]. The Phase I PERFORM study is the only trial at the moment evaluating the safety of FMT from a healthy donor in patients with renal cell carcinoma receiving combination immunotherapy [NCT04163289]. This trial will also prospectively assess the incidence of grade 3 or higher ir-AEs including colitis in renal cell carcinoma patients who will receive FMT before and during combination immunotherapy. 
Table 2 Trials evaluating microbiome and irAEs

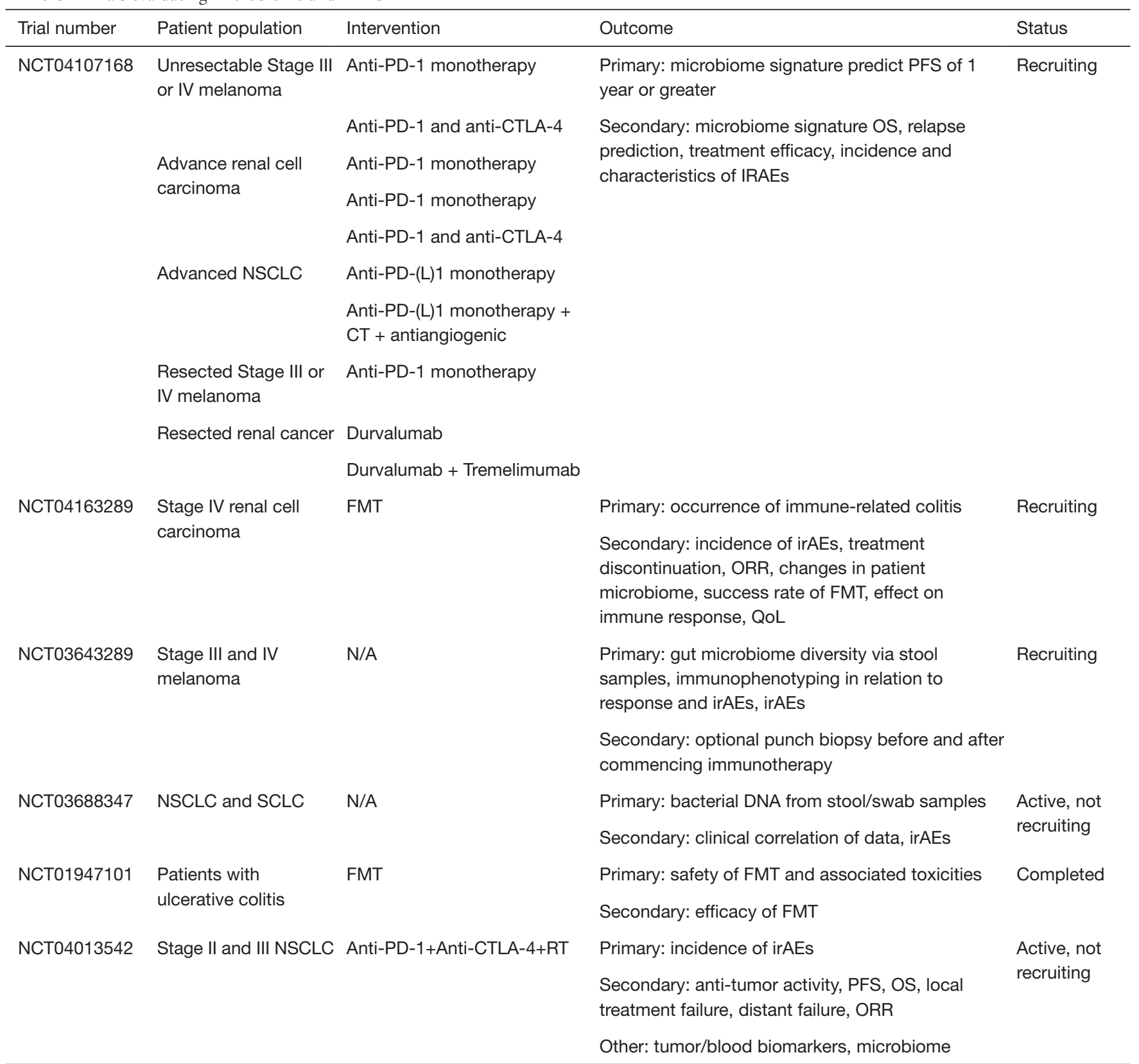

irAEs, inmune-related adverse events; PD-1, programmed death-1 receptor; CTLA-4, cytotoxic T lymphocyte antigen-4; PD-L1, programmed death ligand 1; NSCLC, non-small cell lung cancer; CT, chemotherapy; FMT, fecal microbiota transplantation; N/A, not applicable; ORR, overall response rate; QoL, quality of life; SCLC, small-cell lung cancer; DNA, deoxyribonucleic acid; PFS, progression free survival; OS, overall survival; RT, radiotherapy.

\section{Conclusions}

Preliminary pre-clinical and clinical data suggests a definitive association between gut microbiota and ICPIassociated tumor response and toxicity. Smaller prospective studies and case series also demonstrate a provocative impact of gut microbiome modulation on efficacy and toxicity of ICPIs. As we eagerly await the results of the larger prospective trials that are currently underway to 
evaluate the clinical role of gut microbiome modulation in ICPI therapy, future studies are needed to determine the timing, nature and duration of microbiome modulation to improve the outcomes of patients on ICPI therapy.

\section{Acknowledgments}

The authors would like to thank Ms. Avesta Rastan, scientific designer with Azuravesta design for her services in formulating the figure used in the manuscript.

Funding: None.

\section{Footnote}

Provenance and Peer Review: This article was commissioned by the Guest Editors (Umang Swami and Mohammed M. Milhem) for the series "Cancer Immunotherapy: Recent Advances and Challenges" published in Annals of Translational Medicine. The article has undergone external peer review.

Conflicts of Interest: All authors have completed the ICMJE uniform disclosure form (available at http:// dx.doi.org/10.21037/atm-20-6427). The series "Cancer Immunotherapy: Recent Advances and Challenges" was commissioned by the editorial office without any funding or sponsorship. Dr. DJP reports grants and personal fees from BMS, grants and personal fees from MSD, personal fees from EISAI, personal fees from ROCHE, personal fees from Bayer Healthcare, personal fees from $\mathrm{H} 3 \mathrm{~B}$, personal fees from DaVolterra, personal fees from Falk, personal fees from MiNa Therapeutics, outside the submitted work. Dr. DJP also reports grant support from the NIHR Imperial BRC (Push for Impact 2019 Grant). Dr. SP reports that she is Advisory Board of AstraZeneca, Consultant of G1 therapeutics, and reports research funding from 5 for the Fight. The authors have no other conflicts of interest to declare.

Ethical Statement: The authors are accountable for all aspects of the work in ensuring that questions related to the accuracy or integrity of any part of the work are appropriately investigated and resolved.

Open Access Statement: This is an Open Access article distributed in accordance with the Creative Commons Attribution-NonCommercial-NoDerivs 4.0 International License (CC BY-NC-ND 4.0), which permits the noncommercial replication and distribution of the article with the strict proviso that no changes or edits are made and the original work is properly cited (including links to both the formal publication through the relevant DOI and the license). See: https://creativecommons.org/licenses/by-nc-nd/4.0/.

\section{References}

1. Gandhi L, Rodriguez-Abreu D, Gadgeel S, et al. Pembrolizumab plus Chemotherapy in Metastatic NonSmall-Cell Lung Cancer. N Engl J Med 2018;378:2078-92.

2. Yarchoan M, Hopkins A, Jaffee EM. Tumor Mutational Burden and Response Rate to PD-1 Inhibition. N Engl J Med 2017;377:2500-1.

3. Hills RD Jr, Pontefract BA, Mishcon HR, et al. Gut Microbiome: Profound Implications for Diet and Disease. Nutrients 2019;11:1613.

4. Conlon MA, Bird AR. The impact of diet and lifestyle on gut microbiota and human health. Nutrients 2014;7:17-44.

5. Huang XZ, Gao P, Song YX, et al. Antibiotic use and the efficacy of immune checkpoint inhibitors in cancer patients: a pooled analysis of 2740 cancer patients. Oncoimmunology 2019;8:e1665973.

6. Alexander JL, Kohoutova D, Powell N. Science in Focus: The Microbiome and Cancer Therapy. Clin Oncol (R Coll Radiol) 2019;31:1-4.

7. Das S, Johnson DB. Immune-related adverse events and anti-tumor efficacy of immune checkpoint inhibitors. J Immunother Cancer 2019;7:306.

8. Chaput N, Lepage P, Coutzac C, et al. Baseline gut microbiota predicts clinical response and colitis in metastatic melanoma patients treated with ipilimumab. Ann Oncol 2017;28:1368-79.

9. Gopalakrishnan V, Helmink BA, Spencer CN, et al. The Influence of the Gut Microbiome on Cancer, Immunity, and Cancer Immunotherapy. Cancer Cell 2018;33:570-80.

10. Qin J, Li R, Raes J, et al. A human gut microbial gene catalogue established by metagenomic sequencing. Nature 2010;464:59-65.

11. Gopalakrishnan V, Spencer CN, Nezi L, et al. Gut microbiome modulates response to anti-PD-1 immunotherapy in melanoma patients. Science 2018;359:97-103.

12. Shui L, Yang X, Li J, et al. Gut Microbiome as a Potential Factor for Modulating Resistance to Cancer Immunotherapy. Front Immunol 2020;10:2989.

13. Gong J, Chehrazi-Raffle A, Placencio-Hickok V, et al. The gut microbiome and response to immune checkpoint inhibitors: preclinical and clinical strategies. Clin Transl 
Med 2019;8:9.

14. Sheflin AM, Whitney AK, Weir TL. Cancer-promoting effects of microbial dysbiosis. Curr Oncol Rep 2014;16:406.

15. Sivan A, Corrales L, Hubert N, et al. Commensal Bifidobacterium promotes antitumor immunity and facilitates anti-PD-L1 efficacy. Science 2015;350:1084-9.

16. Zheng Y, Wang T, Tu X, et al. Gut microbiome affects the response to anti-PD-1 immunotherapy in patients with hepatocellular carcinoma. J Immunother Cancer 2019;7:193.

17. Cianci R, Franza L, Schinzari G, et al. The Interplay between Immunity and Microbiota at Intestinal Immunological Niche: The Case of Cancer. Int J Mol Sci 2019;20:501.

18. Maslowski KM. Metabolism at the centre of the hostmicrobe relationship. Clin Exp Immunol 2019;197:193-204.

19. Hussain N, Naeem M, Pinato DJ. Concomitant medications and immune checkpoint inhibitor therapy for cancer: causation or association? Hum Vaccin Immunother 2021;17:55-61.

20. Francino MP. Antibiotics and the Human Gut Microbiome: Dysbioses and Accumulation of Resistances. Front Microbiol 2016;6:1543.

21. Pinato DJ, Howlett S, Ottaviani D, et al. Association of Prior Antibiotic Treatment With Survival and Response to Immune Checkpoint Inhibitor Therapy in Patients With Cancer. JAMA Oncol 2019;5:1774-8.

22. Tinsley N, Zhou C, Tan G, et al. Cumulative Antibiotic Use Significantly Decreases Efficacy of Checkpoint Inhibitors in Patients with Advanced Cancer. Oncologist 2020;25:55-63.

23. Ahmed J, Kumar A, Parikh K, et al. Use of broadspectrum antibiotics impacts outcome in patients treated with immune checkpoint inhibitors. Oncoimmunology 2018;7:e1507670.

24. Kim H, Lee JE, Hong SH, et al. The effect of antibiotics on the clinical outcomes of patients with solid cancers undergoing immune checkpoint inhibitor treatment: a retrospective study. BMC Cancer 2019;19:1100.

25. Derosa L, Hellmann MD, Spaziano M, et al. Negative association of antibiotics on clinical activity of immune checkpoint inhibitors in patients with advanced renal cell and non-small-cell lung cancer. Ann Oncol 2018;29:1437-44.

26. Chalabi M, Cardona A, Nagarkar DR, et al. Efficacy of chemotherapy and atezolizumab in patients with nonsmall-cell lung cancer receiving antibiotics and proton pump inhibitors: pooled post hoc analyses of the OAK and POPLAR trials. Ann Oncol 2020;31:525-31.

27. Zhao S, Gao G, Li W, et al. Antibiotics are associated with attenuated efficacy of anti-PD-1/PD-L1 therapies in Chinese patients with advanced non-small cell lung cancer. Lung Cancer 2019;130:10-7.

28. Schett A, Rothschild SI, Mauti LA, et al. Prognostic impact of the use of antibiotics in patients with advanced non-small cell lung cancer (NSCLC) receiving PD-(L)1 targeting monoclonal antibodies. Ann Oncol 2019;30:ii58.

29. Elkrief A, El Raichani L, Richard C, et al. Antibiotics are associated with decreased progression-free survival of advanced melanoma patients treated with immune checkpoint inhibitors. Oncoimmunology 2019;8:e1568812.

30. Routy B, Gopalakrishnan V, Daillere R, et al. The gut microbiota influences anticancer immunosurveillance and general health. Nat Rev Clin Oncol 2018;15:382-96.

31. Pushalkar S, Hundeyin M, Daley D, et al. The Pancreatic Cancer Microbiome Promotes Oncogenesis by Induction of Innate and Adaptive Immune Suppression. Cancer Discov 2018;8:403-16.

32. Freitag TL, Hartikainen A, Jouhten H, et al. Minor Effect of Antibiotic Pre-treatment on the Engraftment of Donor Microbiota in Fecal Transplantation in Mice. Front Microbiol 2019;10:2685.

33. Naqash AR, Ricciuti B, Owen DH, et al. Outcomes associated with immune-related adverse events in metastatic non-small cell lung cancer treated with nivolumab: a pooled exploratory analysis from a global cohort. Cancer Immunol Immunother 2020;69:1177-87.

34. Inamura K. Roles of microbiota in response to cancer immunotherapy. Semin Cancer Biol 2020;65:164-75.

35. Baiden-Amissah RE, Tuyaerts S. Contribution of Aging, Obesity, and Microbiota on Tumor Immunotherapy Efficacy and Toxicity. Int J Mol Sci 2019;20:3586.

36. Bhandari S, Gill AS, Perez CA, et al. Management of immunotherapy toxicities in older adults. Semin Oncol 2018;45:226-31.

37. Young A, Quant Z, Bluestone JA. The balancing act between cancer immunity and autoimmunity in response to immunotherapy. Cancer Immunol Res 2018;6:1445-52.

38. Schwartz DJ, Rebeck ON, Dantas G. Complex interactions between the microbiome and cancer immune therapy. Crit Rev Clin Lab Sci 2019;56:567-85.

39. Vétizou M, Pitt JM, Dailere R, et al. Anticancer immunotherapy by CTLA-4 blockade relies on the gut microbiota. Science 2015;350:1079-84. 
40. Dasgupta S, Erturk-Hasdemir D, Ochoa-Reparaz J, et al. Plasmacytoid dendritic cells mediate anti-inflammatory responses to a gut commensal molecule via both innate and adaptive mechanisms. Cell Host Microbe 2014;15:413-23.

41. Dubin K, Callagan MK, Ren B, et al. Intestinal microbiome analyses identify melanoma patients at risk for checkpoint-blockade-induced colitis. Nat Commun 2016;7:10391.

42. Simpson R, Batten M, Shanahan E, et al. Intestinal microbiota predict response and toxicities during anti-PD-1/anti-CTLA4 immunotherapy. Pathology 2020;52:S127.

43. Gil-Cruz C, Perez-Shibayama C, De Martin A, et al. Microbiota-derived peptide mimics drive lethal inflammatory cardiomyopathy. Science 2019;366:881-6.

44. Mohiuddin JJ, Chu B, Facciabene A, et al. Association of antibiotic exposure with survival and toxicity in patients

Cite this article as: Naqash AR, Kihn-Alarcón AJ, Stavraka C, Kerrigan K, Maleki Vareki S, Pinato DJ, Puri S. The role of gut microbiome in modulating response to immune checkpoint inhibitor therapy in cancer. Ann Transl Med 2021;9(12):1034. doi: 10.21037/atm-20-6427 with melanoma receiving immunotherapy. J Natl Cancer Inst 2021;113:162-70.

45. Wang F, Yin Q, Chen L, et al. Bifidobacterium can mitigate intestinal immunopathology in the context of CTLA-4 blockade. Proc Natl Acad Sci U S A 2018;115:157-61.

46. Wang Y, Wiesnoski DH, Helmink BA, et al. Fecal microbiota transplantation for refractory immune checkpoint inhibitor-associated colitis. Nat Med 2018;24:1804-8.

47. Helmink BA, Wadud Khan MA, Hermann A, et al. The microbiome, cancer, and cancer therapy. Nat Med 2019;25:377-88.

48. Panebianco C, Andriulli A, Pazienza V.

Pharmacomicrobiomics: exploiting the drug-microbiota interactions in anticancer therapies. Microbiome 2018;6:92. 